DOI: 10.18413/2313-8955-2019-5-1-0-2

\title{
Niklas Padutsch, First report on a 20qh+ heteromorphism characterized by molecular cytogenetics as amplification of D20Z1 sequences
}

Jena University Hospital, Friedrich Schiller University, Institute of Human Genetics, Friedrich-Schiller-Universität Jena, Jena, 07737, Germany Corresponding author: Thomas Liehr(Thomas.Liehr@med.uni-jena.de)

\begin{abstract}
Background: Chromosomal heteromorphisms (CHMs) in human are still understudied. This is partially due to the fact that heterochromatic regions are not well documented in human genome browsers, even though data would be available. Another peculiarity associated with CHMs is that they are routinely seen in cytogenetic analyses, however, considered as being without any clinical meaning. Nonetheless, CHMs are more frequently observed in infertility patients, suggesting very well a potential impact for human genome function. Aim of the study: Since literature is still lacking the exact characterization or complete documentation of numerous $\mathrm{CHMs}$, we aimed to characterize CHMs on chromosome 20 and to characterize whether these result from an amplification of D20Z1 or D20Z2 or both. Materials and methods: An infertile adult and a fetus have been studied by fluorescence in situ hybridization (FISH) with D20Z2 (specific for 20p11.1) and D20Z1 (specific for 20q11.1) probes. Results: Here we prove for the first time that the two different types of CHMs in chromosome 20 are indeed caused by amplification of two different stretches of the alphoid DNA: 20ph+ variants are normally due to enlarged D20Z2-stretches while 20qh+ variants are caused by enlargement of D20Z1-stretches. Conclusions: We have characterized 20qh+ CHM by means of FISH for the first time showing it to result from amplification of D20Z1. This is another puzzle piece for exact characterization and complete documentation of CHMs in human.

Keywords: D20Z1; D20Z2; 20ph+; 20qh+; chromosomal heteromorphisms (CHMs); human

Acknowledgments: The clinical cases were provided by Dr. A. Ovens-Reader Munich, Germany and Dr. I. Bartels, Germany.

Information for citation: Padutsch N, Liehr T. First report on a 20qh+ heteromorphism characterized by molecular cytogenetics as amplification of D20Z1 sequences. Research Results in Biomedicine. 2019;5(1):22-24. DOI: 10.18413/2313-8955-2019-5-1-0-2
\end{abstract}

Introduction. As chromosomal heteromorphisms (CHMs) in human are not well studied or documented, we have recently collected all available data in CHMs in a book [1] and on a webpage [2]. Since the publication of the book in 2014 [1], within only four years alone, in our lab >20 new CHMs were detected and included in the mentioned webpage [2]. This shows that CHMs are clearly understudied, as most of them are only detected in band- ing cytogenetics, but not further followed up by molecular cytogenetics.

As highlighted in [1], DNA-stretches known to be localized as repetitive elements in specific heterochromatic regions of the human genome are still not included in the genome browsers. Data would be available on sequence and average size of these repetitive elements cover. For example, for the alphoid DNA stretch called D20Z1 it is known to be located in cytoband 20q11.1 and to cover about 
$1,020 \mathrm{~kb}$ there [3]. The $171 \mathrm{bp}$ repeats constituting D20Z1 is localized in cytoband 20p11.1 and covers normally about 3,900 kb [4]. These data are not considered in genome browsers e.g. GRCh37 - cytobands 20p11.1 to 201q11.1 are given as $3,800 \mathrm{~kb}$ in size. Also, a genome browser search for 'D20Z1' or 'D20Z2' will not reveal any hit [1].

Here we report the first case with a proven amplification of D20Z1 sequences with banding cytogenetic result $46, X Y, 20 q h+$. Previously only cases with $20 \mathrm{ph}+\mathrm{CHMs}$ and amplification of D20Z2-stretches were reported, which seem to be more frequent, at least in middle European population.

The aim of the study. Here we aimed to characterize CHMs on chromosome 20 and to characterize they were due to amplification of D20Z1 or D20Z2 or both.

Material and methods. Chromosomes were studied from case 1 , an adult studied due to infertility, and from case 2 , a fetus studied here by fluorescence in situ hybridization (FISH) due to advanced maternal age.

Cell culture and chromosomes preparation from blood (case 1) or amniocytes (case 2) were done according to standard procedures [5]. Karyotyping was performed based on GTG-banding [6]. After detection of the variants in chromosome 20, molecular cytogenetic studies were undertaken using a commercially available D20Z2 probe specific for 20p11.1

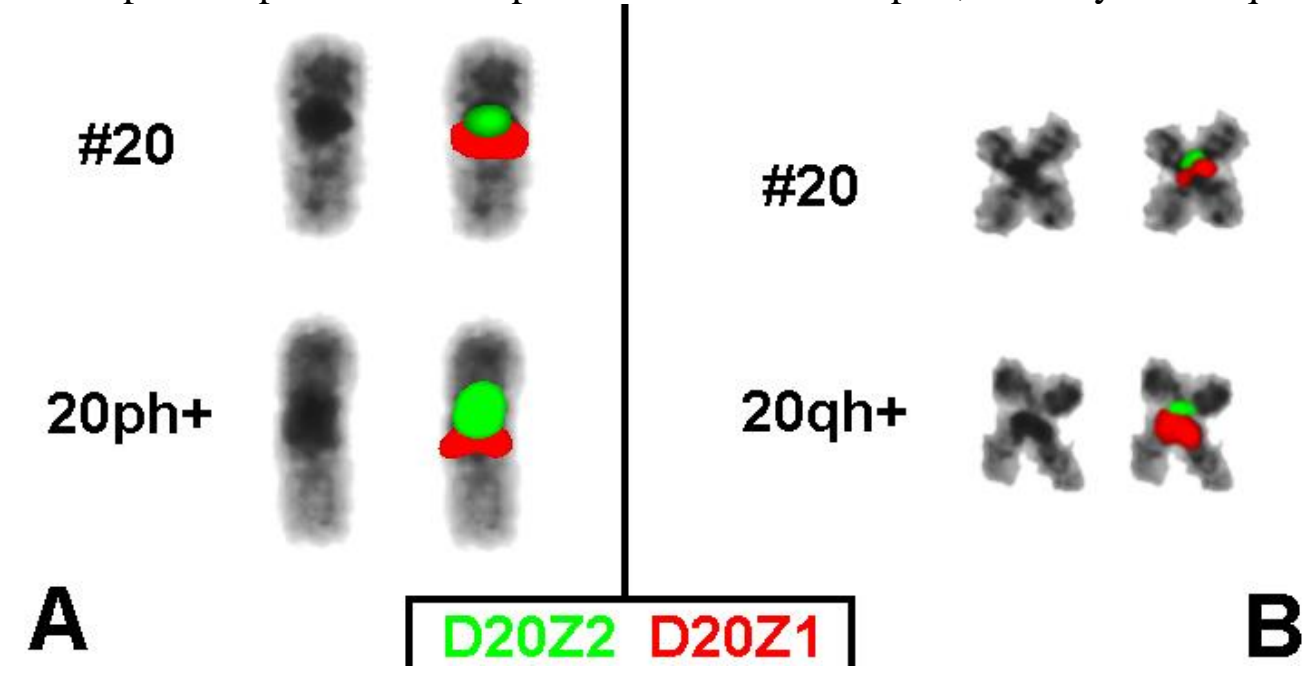

Fig. Results of fluorescence in situ hybridization (FISH) in two cases with centromeric heteromorphisms of chromosome 20 are depicted. In the first column, the inverted DAPI-banding of homologous chromosomes 20 is shown, and the second column shows the same chromosome-pair 20 with FISH- (cep 20, Abbott/Vysis, Wiesbaden, Germany) and the D20Z1 probe specific for 20q11.1, kindly provided by Prof. Mariano Rocchi (Bari, Italy). D20Z2 was commercially available as labelled in SpectrumOrange and D20Z1 was labelled in SpectrumGreen as previously reported. FISH was done according to standard procedures [7], and results were documented using a Zeiss Axioplan microscope equipped with ISIS-software (MetaSystems, Altlussheim, Germany); 20 metaphases were acquired per case.

Results and discussion. As shown in Figure 1, the two probes D20Z2 and D20Z1 cover cytobands $20 \mathrm{p} 11.1$ and 20q11.1, and do not overlap. Also, confirming previous data, FISH-results show in both presented cases that D20Z2 in 20p11.1 normally covers a smaller stretch of DNA than D20Z1 in 20q11.1. In case 1 , the GTG-banding result suggesting a $20 \mathrm{ph}+$ variant was confirmed and the enlarged region of $20 \mathrm{p} 11.1$ is completely covered by D20Z2 specific green FISH signal (similar cases see [1-2]). In case 2 , where a $20 \mathrm{qh}+$ was suggested the result is vice versa, i.e. D20Z1 specific red FISH signal covers the enlarged cytoband 20q11.1. To the best of our knowledge this is the first time it could be demonstrated that $20 \mathrm{qh}+$ variant is due to D20Z2 amplification. Also, it might be of interest that during last 15 years we saw in our lab six $20 \mathrm{ph}+$, but only one $20 \mathrm{qh}+$ variants. 
signals of D20Z1 (red) and D20Z1 (green). In the upper line, normal chromosome 20 (\#20) and in the lower line chromosome 20 with chromosomal heteromorphism are shown.

A) In case 1, a 20ph+ was detected in banding cytogenetics; the enlarged band 20p11.1 was completely covered by green FISH of probe D20Z2.

B) In case 2, a 20qh+ was detected in banding cytogenetics; the enlarged band 20q11.1 was completely covered by red FISH of probe D20Z1.

While the parental origin of the 20qh+ variant could not be sorted out, it is interesting that the $20 \mathrm{ph}+$ variant was seen in a case of unexplained infertility. According to the literature, CHMs are in general more frequently observed in patients with a diagnoses of infertility [8]. A possible explanation for partially unclear data if CHMs are influencing human fertility or not may be, that yet no studies were undertaken to check for a possible 'two-hit-model' effect of CHMs as seen for euchromatic CNVs [910]. It is true that the DNA-stretches constituting CHMs are gene-free, still, a rather disregarded fact is that the alphoid DNA is transcribed into RNA in early embryogenesis [1]. The role and effects thereof are still not studied in detail and far from being understood.

Conclusion. Here we have characterized the yet not molecular studied 20qh+ CHM by means of FISH and could show that it is due to amplification of D20Z1. This is another puzzle piece for exact characterization and complete documentation of CHMs being detectable in human population. Also, only by knowing all variants routinely seen in cytogenetic analyses, their clinical meaning may be uncovered in the future.

No conflict of interest was recorded with respect to this article.

\section{References}

1. Liehr T. Benign \& Pathological Chromosomal Imbalances, Microscopic and Submicroscopic Copy Number Variations (CNVs) in Genetics and Counseling. 1st ed. New York: Academic Press; 2014.

2. Liehr T. 201X. Cases with Heteromorphisms. [Internet]. [cited 2018 Dec 12]. Available from: http://ssmc-tl.com/HMs.html

3. Rocchi M, Baldini A, Archidiacono N, et al. Chromosome-specific subsets of human alphoid DNA identified by a chromosome 2derived clone. Genomics. 1990;8(4):705-709. DOI: https://doi.org/10.1016/0888-7543(90)90258-V

4. Bassi C, Magnani I, Sacchi N, et al. Molecular structure and evolution of DNA sequences located at the alpha satellite boundary of chromosome 20. Gene. 2000;256(1-2):43-50. DOI: https://doi.org/10.1016/S0378-1119(00)00354-1

5. Weise A, Liehr T. Pre- and postnatal diagnostics and research on peripheral blood, bone marrow chorion, amniocytes, and fibroblasts. In: Fluorescence in situ Hybridization (FISH) Application Guide, T Liehr (ed.), 2nd e. Berlin: Springer; 2017:171-180.

6. Kosyakova N, Weise A, Mrasek K, et al. The hierarchically organized splitting of chromosomal bands for all human chromosomes. Mol Cytogenet. 2009;2:4. DOI: 10.1186/17558166-2-4

7. Liehr $\mathrm{T}$, editor. Fluorescence in situ Hybridization (FISH) - Application Guide, 2nd ed. Berlin: Springer; 2017.

8. Kosyakova N, Grigorian A, Liehr T, et al. Heteromorphic variants of chromosome 9. Mol Cytogenet. 2013;6(1):14. DOI: 10.1186/1755-81666-14

9. Girirajan S, Rosenfeld JA, Cooper GM, et al. A recurrent $16 \mathrm{p} 12.1$ microdeletion supports a two-hit model for severe developmental delay. Nat Genet. 2010;42(3):203-209. DOI: 10.1038/ng.534

10. Liehr T. Benign and pathological gain or loss of genetic material- about microscopic and submicroscopic copy number variations (CNVs) in human genetics. Tsitologiia. 2016;58(6):476-477.

\section{Information about the authors}

Niklas Padutsch, BTA; Technician, Jena University Hospital, Friedrich Schiller University, Institute of Human Genetics, Jena, Germany, E-mail: Thomas.Liehr@med.uni-jena.de.

Thomas Liehr, PhD, PD, Head of Lab, Jena University Hospital, Friedrich Schiller University, Institute of Human Genetics, Jena, Germany, E-mail: Thomas.Liehr@med.uni-jena.de, ORCID: 00000003-1672-3054.

Статья поступила в редакцию 15 декабря 2018 г. Receipt date 2018 December 15.

Статья принята к публикации 9 января 2019 г. Accepted for publication 2019 January 9. 